\title{
The effect of disorder on the critical points in the vortex phase diagram of $\mathrm{YBCO}$
}

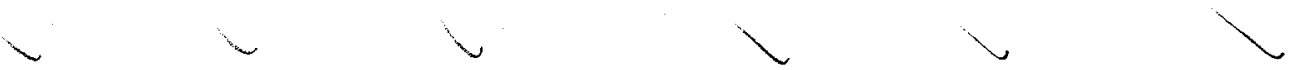 \\ G.W. Crabtree ${ }^{1}$ W.K. Kwok ${ }^{1}$ L.M. Paulius
noTobos ${ }^{1,2,}$ and W.M. Petrean ${ }^{1,2}$ R.J. Olsson $^{1,3}$ G. Karapetrov $^{1}$ V. \\ ${ }^{1}$ Materials Science Division, Argonne National Laboratory, Argonne, IL 60439 \\ ${ }^{2}$ Department of Physics, Western Michigan University, Kalamazoo, MI 49008 \\ ${ }^{3}$ Department of Physics, Michigan State University, East Lansing, MI 48824 \\ ${ }^{4}$ National High Magnetic Field Laboratory, Florida State University, Tallahassee, FL 32306
}

The submitted manuscript has been created by the University of Chicago as Operator of Argonne National Laboratory ("Argonne") under Contract No. W-31-109-ENG-38 with the U.S. Department of Energy. The U.S. Government retains for itself, and others acting on its behalf, a paid-up, nonexclusive, irrevocable worldwide license in said article to reproduce, prepare derivative works, distribute copies to the public, and perform publicly and display publicly, by or on behalf of the Government.

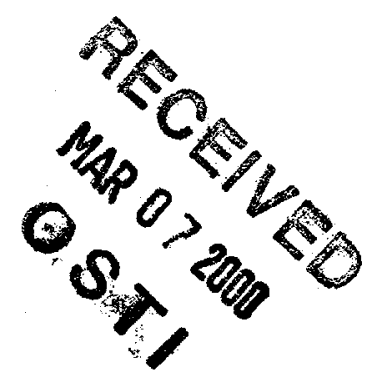

Proceedings of VORTEX 99, Crete, September 18-24, 1999

To appear in Physica C

\section{Distribution:}

\section{1-2. PRS}

3. J.M. Gibson

4. J. Coble

5. File

6. Janice Coble

7-13. Authors

This work is supported by the Division of Materials Sciences, Office of Basic Energy Sciences of DOE, under Contract No. W-31-109-ENG-38, the NSF Science and Technology Center for Superconductivity under contract \#DMR91-20000, Western Michigan University by the US National Science Foundation under contract \#DMR-9624047 and at the National High Magnetic Field Laboratory by the US National Science Foundation under contract \#DMR-9527035. 


\section{DISCLAIMER}

This report was prepared as an account of work sponsored by an agency of the United States Government. Neither the United States Government nor any agency thereof, nor any of their employees, make any warranty, express or implied, or assumes any legal liability or responsibility for the accuracy, completeness, or usefulness of any information, apparatus, product, or process disclosed, or represents that its use would not infringe privately owned rights. Reference herein to any specific commercial product, process, or service by trade name, trademark, manufacturer, or otherwise does not necessarily constitute or imply its endorsement, recommendation, or favoring by the United States Government or any agency thereof. The views and opinions of authors expressed herein do not necessarily state or reflect those of the United States Government or any agency thereof. 


\section{DISCLAIMER}

Portions of this document may be illegible in electronic image products. Images are produced from the best available original document. 


\title{
The effect of disorder on the critical points in the vortex phase diagram of YBCO
}

\author{
G.W. Crabtree' W.K. Kwok' L.M. Paulius ${ }^{1.2}$ A.M. Petrean ${ }^{1.2}$ R.J. Olsson ${ }^{1.3}$ G. Karapetrov' ${ }^{1}$ V. \\ Tobos $^{1.2 .}$ and W.G. Moulton ${ }^{4}$ \\ 'Materials Science Division, Argonne National Laboratory, Argonne, IL 60439 \\ ${ }^{2}$ Department of Physics, Western Michigan University, Kalamazoo, MI 49008 \\ ${ }^{3}$ Department of Physics, Michigan State University, East Lansing, MI 48824 \\ ${ }^{4}$ National High Magnetic Field Laboratory, Florida State University, Tallahassee, FL 32306
}

\begin{abstract}
The submitted manuscript has been created by the University of Chicago as Operator of Argonne National Laboratory ("Argonne") under Contract No. W-31-109-ENG-38 with the U.S. Department of Energy. The U.S. Government retains for itself, and others acting on its behalf, a paid-up, nonexclusive, irrevocable worldwide license in said article to reproduce, prepare derivative works, dis. tribute copies to the public, and perform pubficly and display publicly, by or on behalf of the Government.
\end{abstract}

Proceedings of VORTEX 99, Crete, September 18-24, 1999

To appear in Physica C

\section{Distribution:}

1-2. PRS

3. J.M. Gibson

4. J. Coble

5. File

6. Janice Coble

7-13. Authors

This work is supported by the Division of Materials Sciences, Office of Basic Energy Sciences of DOE, under Contract No. W-31-109-ENG-38, the NSF Science and Technology Center for Superconductivity under contract \#DMR91-20000, Western Michigan University by the US National Science Foundation under contract \#DMR-9624047 and at the National High Magnetic Field Laboratory by the US National Science Foundation under contract \#DMR-9527035. 


\title{
The effect of disorder on the critical points
}

$$
\text { in the vortex phase diagram of } \mathrm{YBCO}
$$

\author{
G. W. Crabtree, ${ }^{1}$ W. K. Kwok, ${ }^{1}$ L. M. Paulius, ${ }^{1,2}$ A. M. Petrean, ${ }^{1,2}$ \\ R. J. Olsson, ${ }^{1,3}$ G. Karapetrov, 1 V. Tobos, ${ }^{1,2}$ and W. G. Moulton ${ }^{4}$
}

1 Materials Science Division, Argonne National Laboratory, Argonne, II 60439 USA

2 Department of Physics, Western Michigan University, Kalamazoo, MI 49008 USA

3 Department of Physics, Michigan State University, East Lansing, MI 48824 USA

4 National High Magnetic Field Laboratory, Florida State University, Tallahassee, FL 32306 USA

\begin{abstract}
The effect of line disorder induced by heavy ion irradiation and of point disorder induced by proton and electron irradiation on the upper and lower critical points in the vortex phase diagram of YBCO is presented. We find that dilute line disorder induces a Bose glass transition at low fields which is replaced at the lower critical point by first order melting at higher fields. Strong pinning point defects raise the lower critical point, while weak pinning point defects have little or no effect on the lower critical point. The upper critical point is lowered by point disorder, but raised by line disorder. First order melting is suppressed by point disorder in two ways, by lowering of the upper critical point only for weak point pins, or by merging of the upper and lower critical points for strong point pins. The differing responses of the upper and lower critical points to line and point disorder can be understood in a picture of transverse and longitudinal spatial fluctuations.
\end{abstract}




\section{Introduction}

The superconducting vortex phase diagram is rich in novel phases and phase transitions. The oldest and best known phase is the vortex lattice, predicted theoretically by Abrikosov ${ }^{1}$ in the mid-1950s. Here the vortices occupy regular positions on a hexagonal lattice, minimizing their mutual interaction energy. In high temperature superconductors thermal energies are large enough to melt the vortex lattice, forming a novel vortex liquid over a large portion of the phase diagram. The vortex lattice melting transition and the liquid phase produce fundamentally new electrodynamic behavior in superconductors. The remarkable effects of thermal disorder on the vortex lattice have been explored in a wide range of innovative experimental and theoretical work. For reviews, see Refs 2-6.

The impact of thermal disorder on the vortex lattice is equaled or exceeded by that of quenched disorder. There is a multitude of disordered solid phases that are expected or observed when random pinning is strong enough to distort or destroy the lattice. These disordered phases are expected to melt in second or higher order phase transitions, unlike the lattice which melts in a thermodynamic first order transition. Experimentally, the effect of disorder is dramatic. Point defects produced by electron ${ }^{7}$ or proton ${ }^{8}$ irradiation and line defects induced by heavy ion irradiation ${ }^{9,10}$ destroy the first order melting transition, shift the irreversibility line, and introduce pinning into the vortex liquid. Theoretically several types of glass states are predicted. Bragg glasses $^{11}$ arise from weak pinning which destroys long range order but maintains topological order, vortex glasse $^{12}$ arise from sufficiently strong point pinning, and Bose glasses ${ }^{13}$ arise from pinning by extended defects like linear heavy ion tracks or planar twin boundaries. The effect of these point and extended defects on the phase diagram is complex, and a coherent picture of the disordered phases has yet to be achieved.

One of the most interesting features of the vortex phase diagram is the unexpected upper and lower critical points where the first order melting line is replaced by a second or higher order transition. These critical points were discovered experimentally and offer a challenge to our basic understanding of vortex behavior. The upper critical point often occurs in the neighborhood of $10 \mathrm{~T}$ in typical clean optimally doped crystals ${ }^{14}$ of $\mathrm{YBCO}$, but 
it is highly sample dependent and occurs as low as $3.5 \mathrm{~T}$ in some nominally clean crystals. ${ }^{15}$ In fully oxygenated crystals, heat capacity experiments ${ }^{16}$ find the upper critical point as high as $26 \mathrm{~T}$ and transport experiments ${ }^{17}$ indicate that it can occur above $30 \mathrm{~T}$. More recently, strong evidence for a lower critical point has been reported from transport, magnetization, and calorimetric experiments. In many nominally clean crystals the first order signature of melting is lost near $T_{\mathfrak{c}}$, at fields ranging from $\sim 1 \mathrm{~T}$ (Refs $18,19,20$ ) to 500 Gauss (Ref 8 ). In twinned crystals with varying degrees of oxygen doping, ${ }^{21,22}$ the lower critical point has been observed at fields of $3-6 \mathrm{~T}$.

The widely varying fields and strong sample dependence reported for the upper and lower critical points suggest that they are associated with disordered phases induced by pinning. However, the existence of more than one type of disorder in many crystals used in previous experiments hides a clear correlation of critical point behavior with pinning defect density and defect geometry. The second or higher order transitions experimentally observed above the upper and below the lower critical points suggest the existence of glassy solid phases. The nature of the glassy phases, and their possible relation to the vortex or Bose glasses, remains unresolved.

In this paper we summarize a series of recent experiments ${ }^{8,10}$ exploring the response of the upper and lower critical points to controlled point and line disorder. We find remarkably systematic behavior which provides new insight into the nature of the critical points and the neighboring disordered phases. We show that line defects produced by heavy ion irradiation induce a Bose glass state below the lower critical point, and that the lower critical point itself moves up monotonically in field with the line defect density. In these crystals, the vortex lattice and Bose glass co-exist in the same sample, separated by the lower critical point. Strong pinning point defects systematically drive the lower critical point up in field, while weak point pins have little or no effect on the low field melting behavior. At high field we show that point defects systematically lower the upper critical point, while line defects raise it. This behavior implies that longitudinal vortex meandering and entanglement play essential roles in the glassy state above the upper critical point. 


\section{Experimental design}

Our experiments on line and point disorder were carried out on high quality, clean, untwinned single crystals which showed strong first order melting. The use of crystals that show first order melting over an extended field range before the addition of pinning disorder ensures that the observed response to added line or point defects is not affected by competition with pre-existing defects. Controlled disorder was added by irradiation with heavy ions at Argonne's ATLAS accelerator, with electrons at Argonne's Electron Microscopy Center, and with protons at Western Michigan University's tandem accelerator. Transport measurements to $8 \mathrm{~T}$ were carried out at Argonne National Laboratory, and to $17 \mathrm{~T}$ at the National High Magnetic Field Laboratory at Florida State University. Comparability among the irradiation doses was ensured by successive irradiation of the same crystal with protons, by successive annealing of the same electron irradiated crystal, or by cleaving a large single crystal into several pieces which were then irradiated with heavy ions to different doses. This procedure prevents sample dependence from affecting the results. As we describe below, our experiments reveal systematic trends in the response of the upper and lower critical points to point and line pinning disorder.

\section{Lower critical point}

\subsection{Line defects}

Figure 1 shows the temperature dependence of the resistivity in several fields of a crystal irradiated with $1.4 \mathrm{GeV} 208 \mathrm{~Pb}^{56+}$ ions to a dose matching field $\mathrm{B}_{\phi}$ of $1000 \mathrm{G}$. The sharp kink in the resistivity typical of first order melting is seen at intermediate fields. At low fields, however, the kink is absent, indicating the existence of a lower critical point where first order melting terminates. The lower critical point is seen clearly in the bottom panel of Figure 1, which shows the temperature derivative of the resistivity at various fields. The sharp peak riding on a broad background in d $\rho / \mathrm{dT}$ (cross-hatched region in Figure 1) is characteristic of first order 
melting. We define the melting temperature $T_{m}$ as the high temperature onset of the peak. in $d \rho / d T$. Simultaneous resistivity and magnetization measurements ${ }^{23}$ indicate that this point coincides with the first nucleation of solid on freezing. The lower and upper critical points are defined by the disappearance of the peak in $\mathrm{d} \rho / \mathrm{dT}$.

Figure 2 shows the $\mathrm{I}-\mathrm{V}$ curves of the $\mathrm{B}_{\phi}=1000 \mathrm{G}$ crystal, taken at $1 \mathrm{~T}$ at the indicated temperatures. The curves show a smooth evolution from linear behavior in the liquid state at high temperature to non-linear behavior at low temperature. The nature of the low temperature solid phase is revealed by the scaling behavior of the I-V curves, shown in the inset. All of the I-V curves collapse onto two curves when plotted in scaled variables, $E / J\left(T-T_{B G}\right)^{v(z-2)} v s J /\left(T-T_{B G}\right)^{3 v}$, where $E$ is the electric field and $J$ the current density. This scaling form is expected for a Bose glass transition, ${ }^{13}$ where the power law divergence at the transition is described by the critical exponents $v$ for the correlation lengths $\left(1_{\perp} \sim 1 /\left(T-T_{B G}\right)^{v}, l_{\|} \sim 1 /\left(T-T_{B G}\right)^{2 v}\right)$ and $z$ for the correlation time $\left(\tau \sim \perp^{\mathrm{z}}\right)$. As the inset shows, scaling is achieved for $v=1.53$ and $z=3.37$, with $\mathrm{T}_{\mathrm{BG}}=89.5 \mathrm{~K}$ at $1 \mathrm{~T}$. These critical exponents are similar to those obtained for other field values in the $\mathrm{B}_{\phi}=1000 \mathrm{G}$ crystal, ${ }^{10}$ and to those obtained for another crystal ${ }^{24}$ with $\mathrm{B}_{\phi}=1 \mathrm{~T}$. They are comparable to the values observed in Bose glasses induced by twin boundaries acting as planar pinning defects, ${ }^{25}$ and to values found in Monte Carlo simulations ${ }^{26}$ of Bose glasses induced by line defects.

The identification of the solid phase below the lower critical point as a Bose glass introduces a new feature into the vortex phase diagram: the transition from a Bose glass to a lattice in the same crystal with increasing vortex density. This phenomenon demonstrates that the effect of disorder can be strongly attenuated with increasing field, in contrast to the second peak effects in BSSCO and YBCO where increasing field enhances disorder. ${ }^{2}$ Physically, the transition from Bose glass to lattice can be understood as a kind of matching field effect. Below and near the matching field, most vortices occupy randomly placed line defect pinning sites. This destroys the translational and orientational order of the lattice and induces the Bose glass. As the field increases, the line pin sites become fully occupied and vortices appear at interstitial positions away from the 
pins. These vortices are free to adjust their positions to minimize the intervortex interaction energy. As the number of interstitial vortices grows with field, the pinning energy favoring the glass saturates while the interaction energy favoring the lattice continues to increase. At the lower critical point the lattice is sufficiently well formed to produce first order melting.

Our data at $\mathrm{B}_{\phi}=50 \mathrm{G}, 500 \mathrm{G}$, and $1000 \mathrm{G}$ (see the inset of Figure 5) show that the position of the lower critical point increases monotonically with the line defect density, occurring at approximately 40 times the matching field. ${ }^{10}$ This rough scaling provides an estimate of the lattice disorder required to destroy first order melting. In the presence of the line disorder in our experiments, displacement of about $2.5 \%$ of the vortices from their equilibrium positions is sufficient to destroy first order melting. Alternatively, the scaling of the lower critical point and the matching field can be viewed as measuring the minimum correlation length needed for first order melting. When the correlation length exceeds approximately $(40)^{1 / 2}$ vortex spacings, first order melting first appears.

The confluence of first order lattice melting and second order Bose glass melting at the lower critical point implies a new phase line separating the lattice from the Bose glass in the vortex solid. This line should terminate at or near the lower critical point, similar to the behavior of the "second peak" and fishtail lines near the upper critical point. The location of the new line in the phase diagram will indicate the relative stability of the lattice and Bose glass phases at low temperature.

\subsection{Point defects}

The effect of point defects on the melting transition is shown in Figure 3, where the temperature derivative of the resistivity is plotted for fields up to $8 \mathrm{~T}$ for the unirradiated crystal and after irradiation with $9 \mathrm{MeV}$ protons to doses of $1 \times 10^{15} \mathrm{p} / \mathrm{cm}^{2}$ and $1.5 \times 10^{15} \mathrm{p} / \mathrm{cm}^{2}$. The inset shows the behavior at low field for the unirradiated crystal. This crystal shows the first order melting peak at fields below $500 \mathrm{G}$, the lowest value yet reported for the lower critical point. ${ }^{8}$ With successive irradiations, the lower critical point rises in field, 
qualitatively similar to the effect of line defects. The disappearance of first order melting at low field implies that the lattice is destroyed by pinning disorder in this region. However, unlike for line defects, the nature of the disordered state below the lower critical point is not clear. Although it is reasonable to expect a vortex glass there, we do not find the predicted theoretical signatures of this glassy state for these irradiation doses. At considerably higher proton doses, above $3 \times 1016 \mathrm{p} / \mathrm{cm}^{2}$, where the first order transition is suppressed at all fields, indications of a vortex glass state can be seen. ${ }^{27}$ The region of stability of the vortex glass in the phase diagram, its dependence on the strength and density of the point pinning defects, and the possibility that random point disorder might destroy first order melting without producing a vortex glass are major unresolved issues.

The effect of point defects on the lower critical point is not universal. Although proton induced point defects raise the lower critical point as shown in Figure 3, point defects due to electron irradiation and oxygen vacancies have little effect. Irradiation with $1 \mathrm{MeV}$ electrons at doses up to $1 \times 10^{19} \mathrm{e} / \mathrm{cm}^{2}$ does not measurably shift the lower critical point, ${ }^{8}$ although the upper critical point is dramatically lowered as described below. Similarly, systematic studies ${ }^{17}$ of the melting line as a function of oxygen stoichiometry show large effects on the upper critical point but little if any effect on the lower critical point.

The reason for this seemingly contradictory behavior of point defects on the lower critical point lies in their differing pinning strengths. Comparisons of pinning strength show that proton- induced defects pin the vortex liquid more effectively than electron-induced defects even when the electron-induced defect density significantly exceeds the proton-induced density. ${ }^{2728}$ This difference in pinning strength may arise from a significant difference in defect size. Protons produce not only displaced atoms but also cluster defects ${ }^{29}$ which are larger than the defects produced by electron irradiation. ${ }^{28}$ The larger size of proton induced defects is likely to make them effective pinners close to $T_{c}$ where they better match the diverging superconducting coherence length and vortex core size. This larger pinning strength near $\mathrm{T}_{\mathrm{c}}$ is crucial to disordering the lattice at the lower critical point. Near $T_{c}$ the intervortex interaction energy decreases with the decrease in vortex density as the field is lowered, and the pinning energy goes to zero with the condensation energy. Lower critical points due to pinning disorder occur only if the pinning strength near $T_{c}$ dominates the vortex interaction strength. Weak 
pinners like electron induced point defects and oxygen vacancies are evidently not strong enough to disorder the lattice at high temperature, while strong pinners like proton-induced point defects and heavy ion tracks overcome the vortex interaction energy near $\mathrm{T}_{\mathfrak{c}}$ and disorder the lattice enough to destroy first order melting.

\section{Upper critical point}

\subsection{Point defects}

Figure 4 summarizes the effect of electron- and proton-induced point defects on the melting line. The inset shows the melting line before electron irradiation, after irradiation with $1 \mathrm{MeV}$ electrons to a dose of $1 \times 1019$ $\mathrm{e} / \mathrm{cm}^{2}$, and after successive anneals at temperatures between $100^{\circ} \mathrm{C}$ and $240^{\circ} \mathrm{C}$ as indicated in the caption. The initial electron irradiation dramatically lowers the upper critical point. Successive anneals gradually remove the point defects and the upper critical point recovers as shown, with no apparent effect on the low field region. The annealing measurements show that increasing point defect density due to electron irradiation systematically lowers the upper critical point. A similar lowering of the upper critical point by proton-induced defects can be seen in $\mathrm{d} \rho / \mathrm{dT}$ plotted in Figure 3, and in the phase diagram derived from this data shown in the main panel of Figure 4. In this crystal the upper critical point is above the highest measuring field, $8 \mathrm{~T}$, before irradiation and moves down to $\sim 7 \mathrm{~T}$ after a dose of $1.5 \times 10^{15} \mathrm{p} / \mathrm{cm}^{2}$ as shown in Figure 4. The widths and heights of the peaks in $\mathrm{d} \rho / \mathrm{dT}$ indicate that the upper critical point moves down in field with increasing proton-induced point defect density.

Figure 4 shows an important feature of the effect of point disorder on melting. The first order transition can disappear in at least two ways. In electron irradiated samples, the upper critical point moves down with point disorder, while the lower critical point remains virtually unaffected. Vortex disorder creeps down from high fields, randomizing the lattice and destroying first order melting. In contrast, proton-induced disorder simultaneously drives the upper critical point down and the lower critical point up. The vortex lattice and first 
order melting are squeezed out by the merging of the two critical points. The existence of two disordered states in the case of proton-induced point disorder raises the interesting question of whether and in what ways they differ. A quantitative theoretical picture of these point disordered states is not yet available.

\subsection{Line defects}

The effect of random line defects on melting is strongest at low fields, where the first order lattice melting transition is transformed into a higher order Bose glass transition. With increasing field, it is reasonable to expect that the impact of the line defects diminishes, as the pinned vortices are heavily outnumbered by interstitial vortices. Such behavior is seen in Figure 5, which shows the resistive phase diagram derived from data like that in Figure 1 for the unirradiated crystal and for the $B_{\phi}=1000 \mathrm{G}$ crystal. Just above the lower critical point, the first order melting transition occurs at the same temperature and field in both crystals, suggesting that line defects have no noticeable effect at these fields. Remarkably, at higher field a new effect of line defects becomes visible. The first order melting transition is raised in temperature and field by line defects, opposite to the effect of point defects which depress the first order melting point. Moreover, the upper critical point itself is raised by line defects, extending first order melting to higher fields. The enhancement of the upper critical point is shown in detail in Figure 1, where the peak in $\mathrm{d} \rho / \mathrm{dT}$ can be seen up to $11 \mathrm{~T}$ in the irradiated crystal but only to $9 \mathrm{~T}$ in the unirradiated one.

These unexpected high field effects of line defects cannot be due to their random lateral placement disordering the lattice, since that effect is lost above the lower critical point. Rather, the origin of the high field effects is the line character of the defects. Line defects tend to straighten vortices, reducing their lateral wandering. The enhancement of the first order melting temperature and the upper critical point indicates that vortex wandering and entanglement are essential features of the liquid and disordered states at high field. This conclusion is confirmed by the response of the high field liquid and disordered states to point defects as shown in Figures 3 and 4. Point defects promote vortex wandering and entanglement, and they depress both the first 
order melting transition and the upper critical point. This conclusion is further confirmed by the behavior of fully oxygenated crystals with little oxygen vacancy disorder, where the upper critical point is enhanced to above $26 \mathrm{~T}^{16}$ or $30 \mathrm{~T} .^{17}$

Our results suggest a qualitative difference between the low field and high field disordered states in the presence of point defects. The low field disordered state is stabilized by both point and line defects, as if the primary form of disorder were the random lateral displacement of the vortices by defects. The high field disordered state, however, is stabilized by point defect but destabilized by line defects. This implies that not only lateral disorder but also longitudinal disorder in the form of vortex wandering and entanglement plays an important role in the high field behavior. The importance of lateral randomization in the low field disordered vortex structure and of longitudinal wandering and entanglement in the high field disordered structure may be key features distinguishing the two disordered states.

In summary, we present new systematic data revealing the separate roles of point and line disorder on first order melting and the upper and lower critical points of YBCO. Line defects and strong point pins induce a well defined lower critical point separating a disordered vortex state at lower field from a lattice at higher field. Critical scaling of the I-V curves identifies the low field disordered state in the presence of line defects as a Bose glass. The occurrence of a Bose glass and a vortex lattice in the same crystal introduces a new level of complexity and richness to the vortex phase diagram. In the case of point defects, neither the low field nor the high field disordered states can be identified as a vortex glass, and their natures remain mysterious. Remarkably, line defects raise the upper critical point even though their disordering effect on the lateral vortex positions is lost at the lower critical point. This implies that longitudinal vortex wandering and entanglement are important factors in the disordered state above the upper critical point.

Work at Argonne National Laboratory was supported by the US Department of Energy, BES-Materials Sciences under contract \#W-31-109-ENG-38 (GWC, WKK, LMP, AMP, RJO) and by the NSF Science and Technology Center for Superconductivity under contract \#DMR91-20000 (GK), at Western Michigan University by the US National Science Foundation under contract \#DMR-9624047 (LMP, AMP, VT) and at the 
National High Magnetic Field Laboratory by the US National Science Foundation under contract \#DMR9527035 (WGM).

\section{References}

'A. A. Abrikosov, Zh. Eksp. Teor. Fiz. 32, (1957) 1442-1452 [Sov. Phys. JETP 5, (1957) 1174-1182].

${ }^{2}$ G. W. Crabtree, W. K. Kwok, U. Welp, D. Lopez, and J. A. Fendrich, in Physics and Materials Science of Vortex States, Flux Pinning, and Dynamics, Proceedings of the NATO ASI, Kusadasi, Turkey, 1998, (R. Kossowsky, S. Bose, V. Pan, and Z. Durusoy, eds) Kluwer (Dordrecht), 1999, p. 357.

${ }^{3}$ George W. Crabtree and David R. Nelson, Physics Today 50 (4), 38-45 (1997).

${ }^{4}$ G. W. Crabtree, W. K. Kwok, U. Welp, J. A. Fendrich and B. W. Veal, J. Low Temp. Phys. 105, 1073-1082 (1996).

${ }^{5}$ G. Blatter, M. V. Feigel'man, V. B. Geshkenbein, A. I. Larkin, and V. M. Vinokur, Rev. Mod. Phys. 66, 1125 -1388 (1994).

${ }^{6}$ G. W. Crabtree, D. Lopez, W. K. Kwok, H. Safar and L. M. Paulius, Proceedings of MOS 99, Stockholm, Sweden, July 28-Aug 4, 1999, Journal of Low Temperature Physics, In Press.

' J. A. Fendrich, W. K. Kwok, J. Giapintzakis, C. J. van der Beek, V. M. Vinokur, S. Fleshler, U. Welp, H. K. Viswanathan, and G. W. Crabtree, Phys. Rev. Lett. 74 (1995) 1210-1213.

${ }^{8}$ L. M. Paulius, W. -K. Kwok, R. J. Olsson, A. M. Petrean, V. Tobos, J. A. Fendrich, G. W. Crabtree, C. A. Burns, and S. Ferguson (1999) pre print.

${ }^{9}$ L. M. Paulius, J. A. Fendrich, W.-K. Kwok, A. E. Koshelev, V. M. Vinokur, and G. W. Crabtree and B. G. Glagola, Phys Rev B 56 (1997) 913.

${ }^{10}$ W. -K. Kwok, R. J. Olsson, G. Karapetrov, L. M. Paulius, W. G. Moulton, D. J. Hoffman, and G. W. Crabtree (1999) pre-print.

${ }^{11}$ P. Le Doussal and T. Giamarchi, Phys Rev B 57 (1998) 1135.

${ }^{12}$ D. S. Fisher, M. P. A. Fisher, and D. A. Huse

Phys. Rev. B 43 (1991) 130-159.

${ }^{13}$ D. R. Nelson and V. M. Vinokur, Phys Rev B 48 (1993) 13060.

${ }^{14}$ H. Safar, P. L. Gammel, D. A. Huse, D. J. Bishop, W. C. Lee, J. Giapintzakis, D. M. Ginsberg, Phys. Rev. Lett. 70 (1993) 3800-3803.

${ }^{15}$ D. López, L. Krusin-Elbaum, H. Safar, E. Righi, F. de la Cruz, S. Grigera, C. Feild, W. K. Kwok, L. Paulius, and G. W. Crabtree, Phys Rev. Lett. 80 (1998) 1070.

${ }^{16}$ F. Bouquet, C. Marcenat, R. Calemczuk, A. Erb, A. Junod, M. Roulin, U. Welp, W. -K. Kwok, G. W. Crabtree, N. E. Phillips, R. A. Fisher and A. Schilling, in Physics and Materials Science of Vortex States, Flux Pinning, and Dynamics, Proceedings of the NATO ASI, Kusadasi, Turkey, 1998, (R. Kossowsky, S. Bose, V. Pan, and Z. Durusoy, eds) Kluwer (Dordrecht), 1999, p. 743.

${ }^{17}$ T. Nishizaki, K. Shibata, T. Naito, M. Maki, and N. Kobayashi, Proceedings of MOS 99, Stockholm, Sweden, July 28-Aug 4, 1999, Journal of Low Temperature Physics, In Press.

${ }^{18}$ U. Welp, J. A. Fendrich, W. -K. Kwok, G. W. Crabtree, and B. W. Veal, Phys. Rev. Lett. 76, (1996) 48094013.

${ }^{19}$ A. Schilling, R. A. Fisher, N. E. Phillips, U. Welp, D. Dasgupta, W. -K. Kwok, and G. W. Crabtree, Nature (London) 382, (1996) 791-793.

${ }^{20}$ M. Willemin, A. Schilling, H. Keller, C. Rossel, J. Hofer, U. Welp, W. K. Kwok, R. J. Olsson, and G. W. Crabtree. Phys. Rev. Lett. 81 (1998) 4236.

${ }^{21}$ M. Roulin, A. Junod, A. Erb and E. Walker, Phys. Rev. Lett 80 (1998) 1722. 
${ }^{22}$ B. Revaz, A. Junod, and A. Erb, Phys Rev B 58 (1998) 11153.

${ }^{23}$ J. A. Fendrich, U. Welp, W. -K. Kwok, A. Koshelev, G. W. Crabtree and B. W. Veal, Phys. Rev. Lett. 77 (1996) 2073-2077.

${ }^{24}$ R. J. Olsson, W. -K. Kwok, G. W. Crabtree, (1999) unpublished.

${ }^{25}$ S. A. Grigera, E. Morré, E. Osquiguil, C. Balseiro, G. Nieva, and F. de la Cruz, Phys. Rev. Lett. 81 (1998) 2348.

${ }^{26} \mathrm{~J}$. Lidmar and M. Wallin, (1998) cond mat/9812342.

${ }^{27}$ A. M. Petrean, L. M. Paulius, W. -K. Kwok, J. A. Fendrich and G. W. Crabtree (1999) pre-print.

${ }^{28}$ J. Giapintzakis, W. C. Lee, J. P. Rice, D. M. Ginsberg, I. M. Robertson, R. Wheeler, M. A. Kirk, and M.-O. Ruault, Phys. Rev. B 45, (1992) 10677-10683.

${ }^{29}$ M. A. Kirk and H. W. Weber, Studies of High Temperature Superconductors, Vol. 10, A. Narlikar, ed. (Nova Science, 1992), 243.

\section{Figure Captions}

Figure 1 Top panel: resistivity of untwinned YBCO irradiated with $1.4 \mathrm{GeV} 208 \mathrm{~Pb} 56+$ ions to a dose matching field $B_{\phi}$ of $1000 \mathrm{G}$. Middle panel: temperature derivative of the resistivity, $d \rho / d T$ before irradiation. Lower panel: temperature derivative of the resistivity, $d \rho / d \mathrm{~T}$, after irradiation to a matching field of $1000 \mathrm{G}$. The cross-hatched peak defines first order melting.

Figure $2 \mathrm{I}-\mathrm{V}$ curves of the $\mathrm{B}_{\phi}=1000 \mathrm{G}$ crystal, showing a smooth transition from linear to non-linear as the temperature decreases. The inset shows the scaling expected for a Bose glass.

Figure $3 \mathrm{~d} / \mathrm{dT}$ for an untwinned crystal of YBCO before and after $9 \mathrm{MeV}$ proton irradiation to doses of $1 \times 10^{15} \mathrm{p} / \mathrm{cm}^{2}$ and $1.5 \times 10^{15} \mathrm{p} / \mathrm{cm}^{2}$. The inset shows the behavior at low fields, where the first order melting peak persists to below $500 \mathrm{G}$. The temperature axis is normalized to $T_{\text {co }}$, the zero field resistive transition in the unirradiated crystal.

Figure 4 The phase diagram of the proton irradiated YBCO crystal of Figure 3. The inset shows the phase diagram of an untwinned crystal irradiated with $1 \mathrm{MeV}$ electrons to a dose of $1 \times 10^{19} \mathrm{e} / \mathrm{cm}^{2}$, followed by

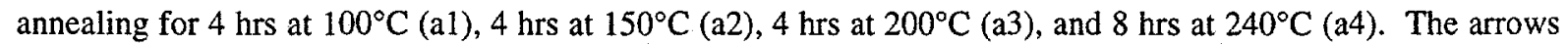
mark the positions of the upper critical point after each anneal. The temperature axis is normalized to $\mathrm{T}_{\text {co, }}$ the zero field resistive transition in the unirradiated crystal.

Figure $5 \mathrm{H}$-T phase diagram for the untwinned crystal of Figure 1 before and after irradiation with $1.4 \mathrm{GeV}$ $208 \mathrm{~Pb}^{56+}$ ions to a dose matching field $\mathrm{B}_{\phi}$ of $1000 \mathrm{G}$. The points above the upper critical points are the irreversibility lines determined from a resistivity criteria of $0.01 \mu \Omega-\mathrm{cm}$. The inset shows the position of the lower critical point after heavy ion irradiation to matching fields $B_{\phi}$ of $50 \mathrm{G}, 500 \mathrm{G}$, and $1000 \mathrm{G}$. The temperature axis of the inset is normalized to $T_{\mathrm{co}}$, the zero field resistive transition in the unirradiated crystal. 


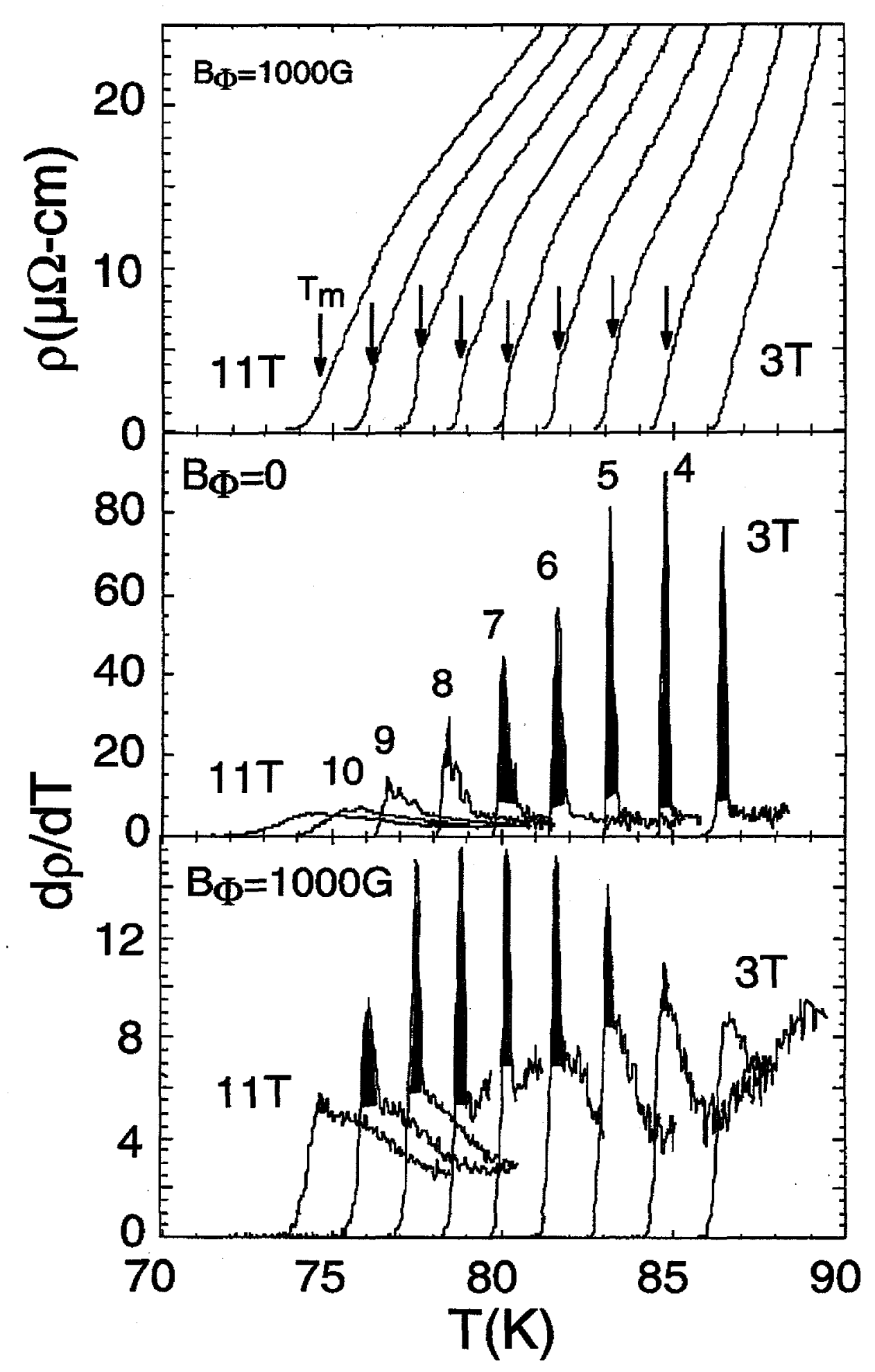

Figure 1 


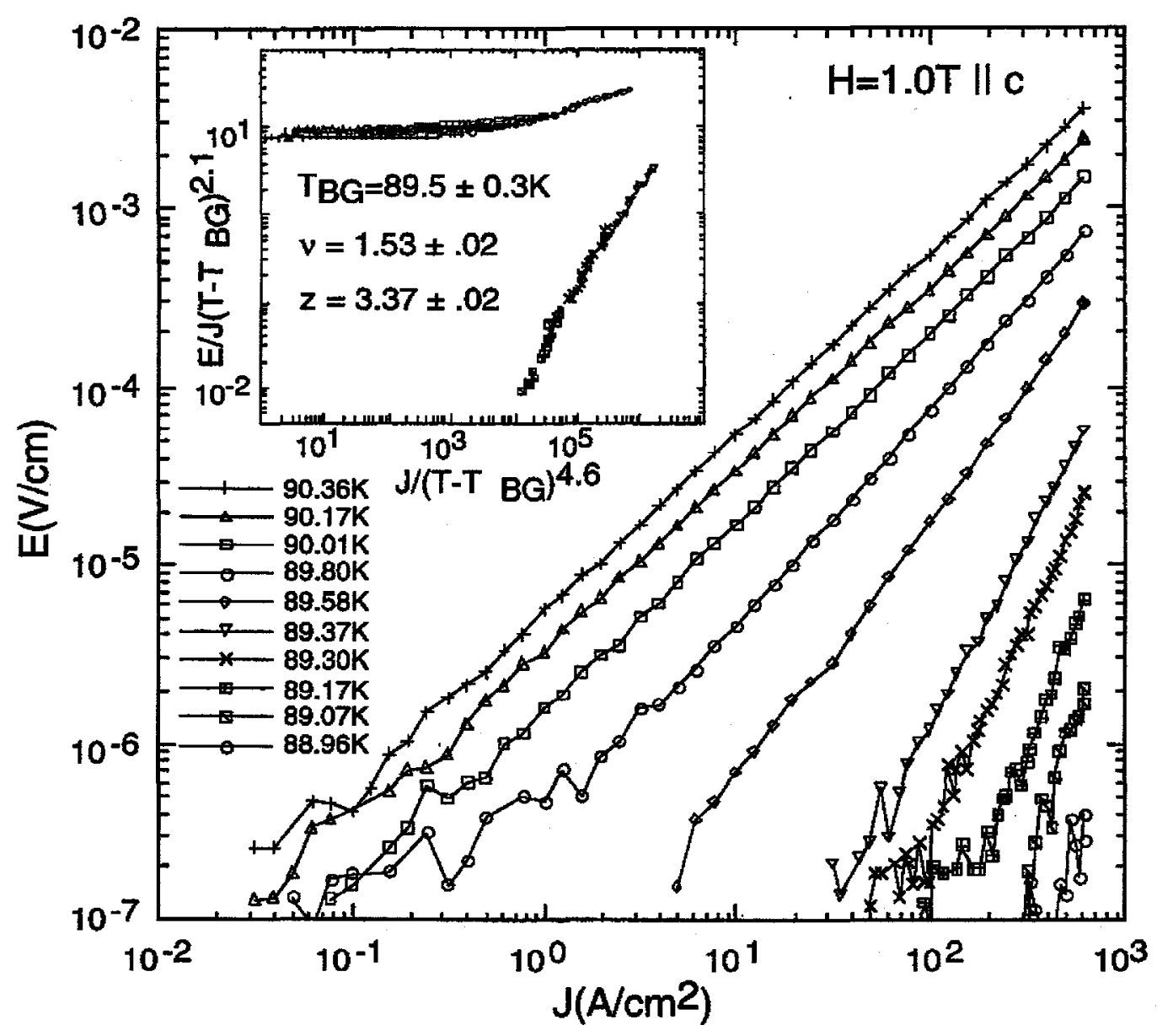

Figure 2 


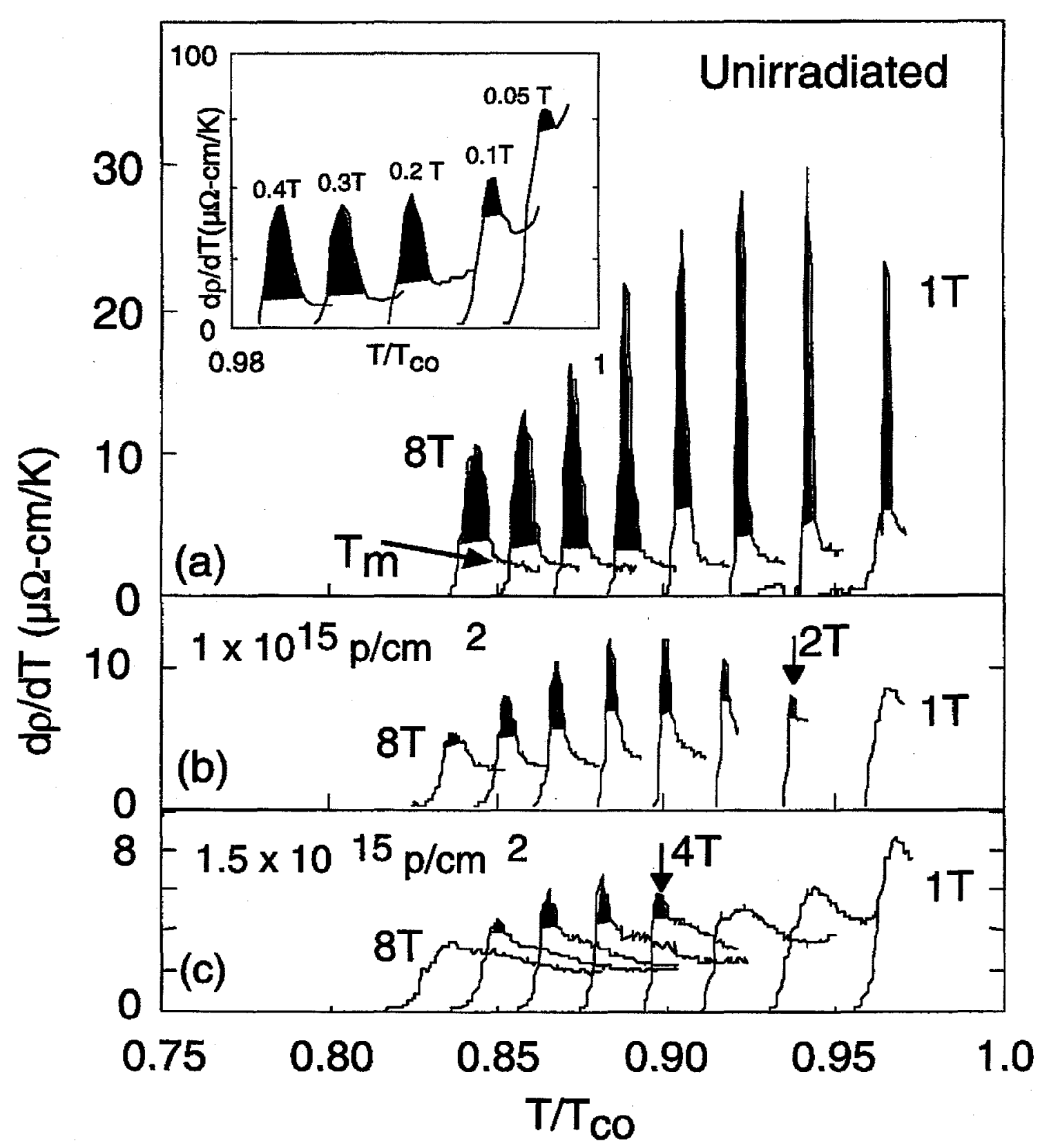

Fiqure 3 


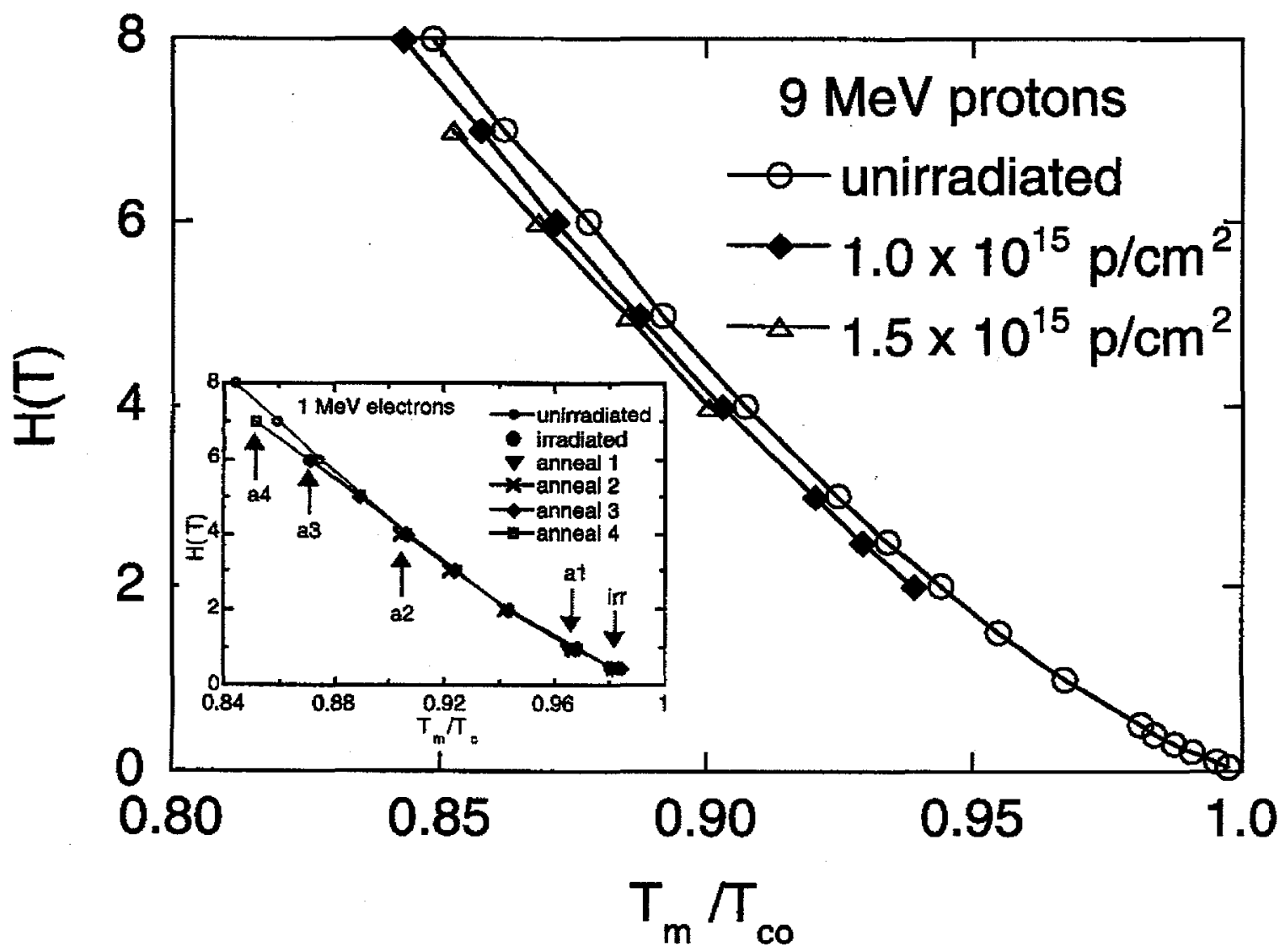

Figure 4 


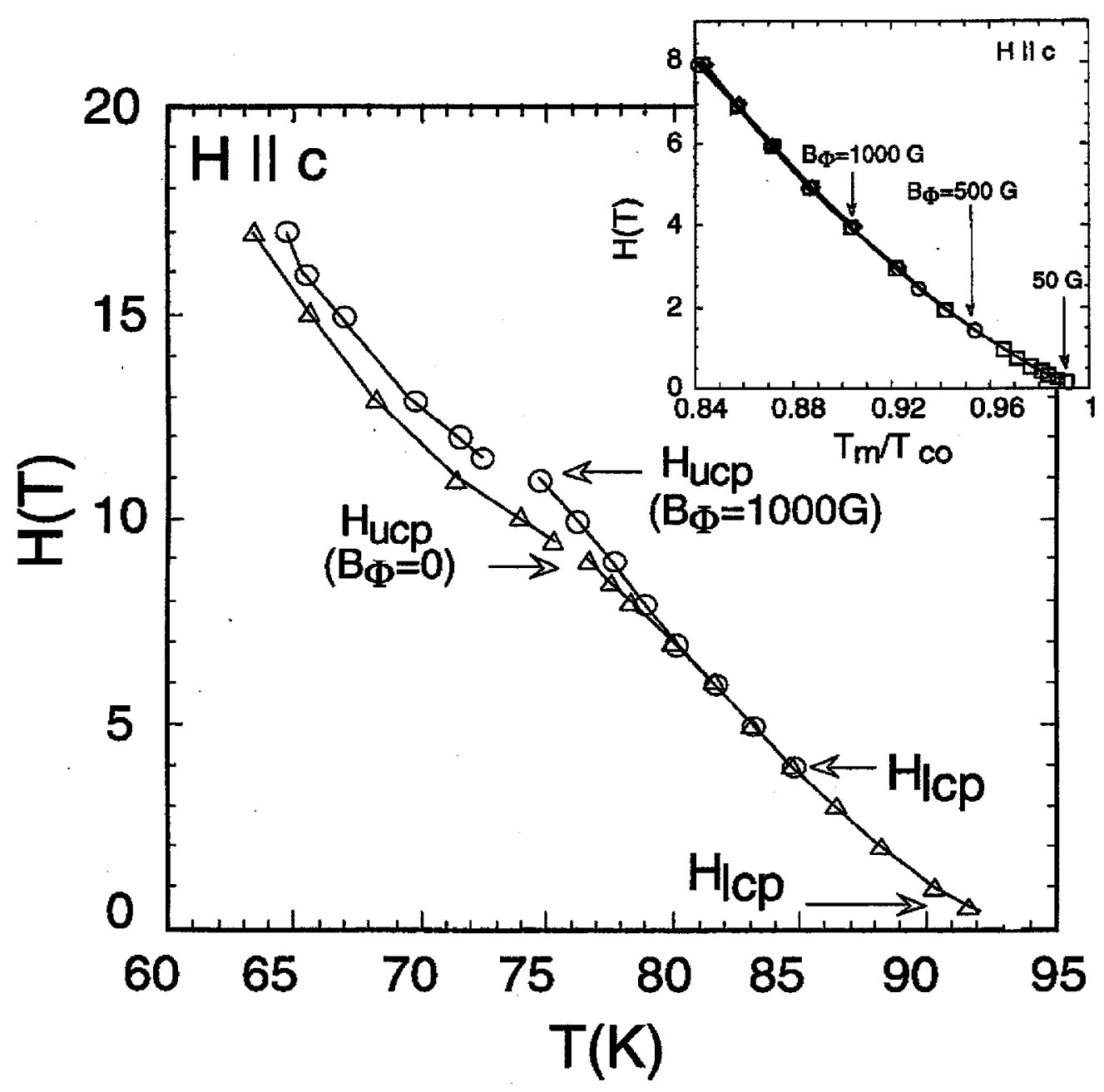

Figure 5 